\title{
ENFERMERÍA TRANSCULTURAL, ELEMENTO VITAL DE SEGURIDAD DEL PACIENTE
}

Mtra. Ma. Teresa Perez Rodriquez"

-Profesora Titular A Secretaria de Planeación y Evalucción. ENEO-UNAM

Podemos afirmar que el cuidado que nos identifica en la perspectiva de la enfermería transcultural es un cuidado reciproco, interactivo e integrador, en el cual las experiencias vividas y trascendentes comprenden la interacción entre personas totales con sus sentimientos, pensamientos y expresiones.

Interacción reciproca significa un encuentro de seres humanos diferentes, con sabidurias y mentalidades que cada uno ofrece en el encuentro, donde la persona del enfermo ofrece su ser total, sus conocimientos, sus observaciones y percepciones, consciente de que es un ser humano susceptible y vulnerable. El ambiente del cuidado debe favorecer la prioridad, el pudor y la unicidad de cada ser humano, sujeto reconocido del encuentro transcultural.

Gracias a las actividades auténticas de la Enfermera (o), conocimiento, reflexión, técnicas del cuidado y momentos especiales del mismo, se da esa interacción, en la que la persona ofrece su confianza, asi como su interés porque se le reestructure y reintegre como persona digna de apoyo, consideración, respeto y afecto.

PALABRAS CLAVE: Enfermerla Transcultural, persona, apoyo.

\section{Abstract}

The care that identifies us in Transcultural Nursing is a reciprocal, interactive and integral care, in which the transcendent experiences are comprehended by the interaction among people, their feelings, their thoughts and expressions.

Reciprocal interaction is a gathering of different human beings, in which they share their own wisdams and mentalities. The patient completely offers his knowledge, observations and perceptions, conscious that he is a capable and vulnerable individual. The health care environment must enhance priority, modesty and the uniqueness of each patient, who is recognized as the subject of transcultural interaction.

Thanks to the authentic activities that nurses take on: knowledge, reflection, health care techniques and special moments of thase duties, it is possible to reach an interaction. In this interaction the patient offers his trust, as well as his interest in that he is restructured and restored as a person worthy of support, consideration, respect and affection.

KEY WORDS: Transcultural Nursing, person, support.

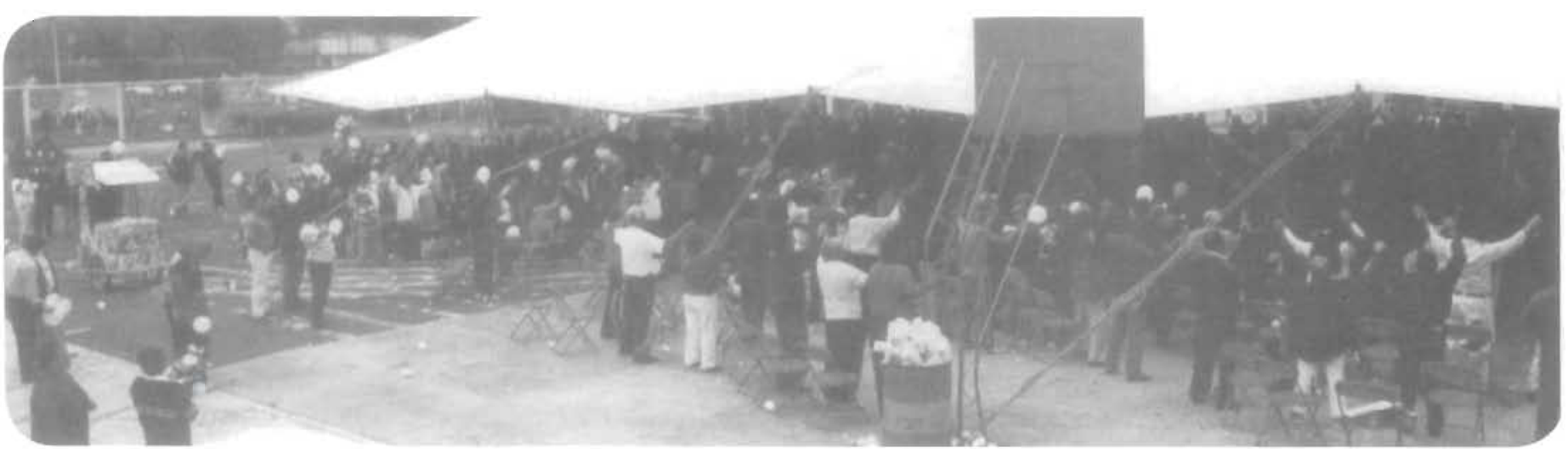




\section{INTRODUCCIÓN}

Las preguntas en que se centra esta presentación se refieren a LPorqué las Enfermeras deben preocuparse acerca de un cuidado culturalmente competente? y iCuáles son los conceptos básicos que entraña una práctica de. Enfermería transcultural?

En primer lugar es necesario recordar que nuestro pais se caracteriza por una diversidad de grupos sociales, con grandes riquezas culturales que los diferencian entre si y de otros paises. Por otro lado la migración interna que naturalmente ha ocurrido en la población mexicana, a través de su historia, ha ocasionado que dichos grupos confluyan en determinados lugares para trabajar, compartir y adoptar costumbres al recibir los servicios básicos asi como de atención a su salud. Este proceso ocasiona desconcierț y una vulnerabilidad cultural de grupos amplios de población que, al arribar a la ciudad tienen que modificar sus esquemas previos al enfrentar condiciones de pobreza extrema, mayor morbilidad y mortalidad por patologías del subdesarrollo, que se asocia a las precarias condiciones de la vivienda, los

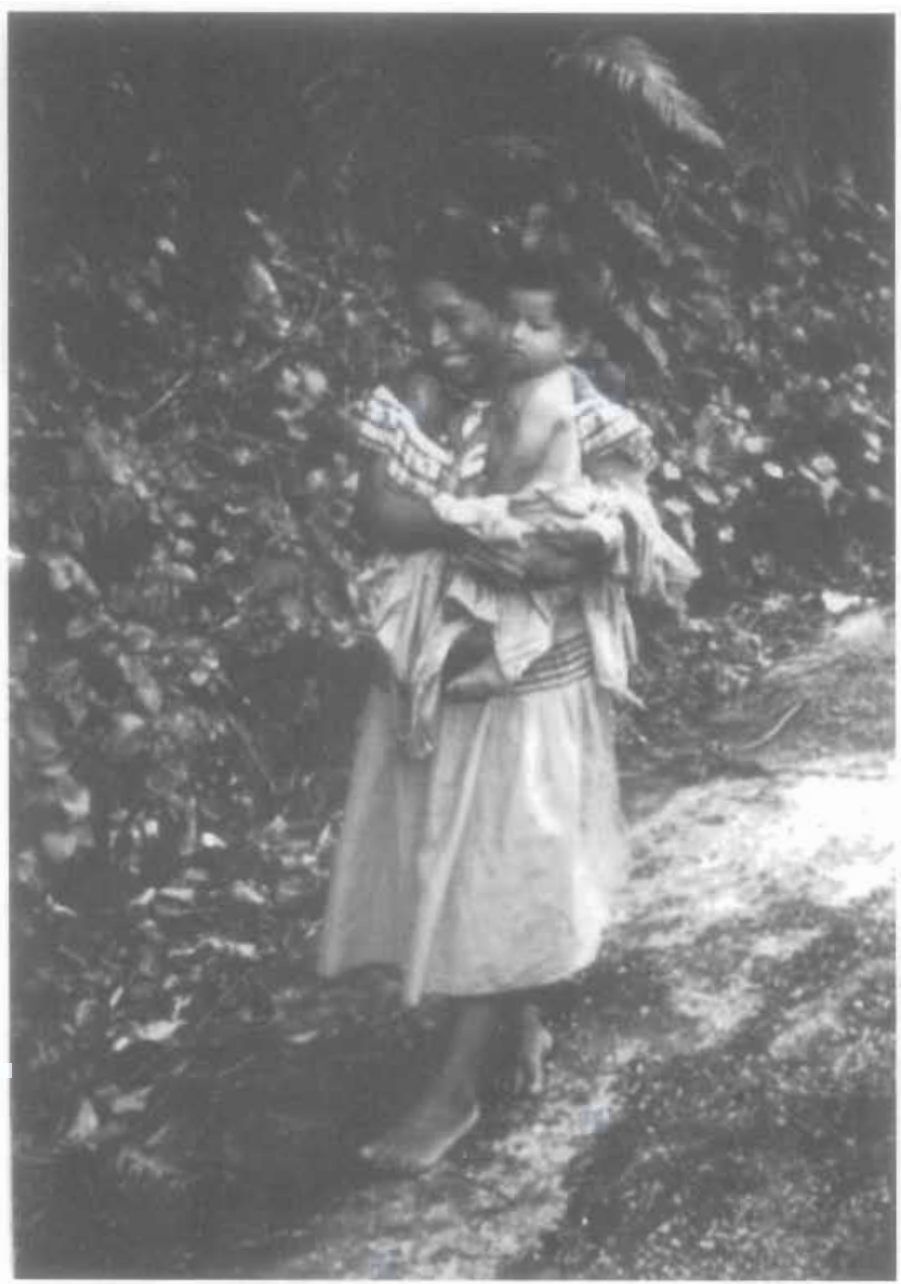

bajos ingresos y la falta de disponibilidad de los servicios básicos, con la consecuente sustitución de sus modos de vida propios, por otros que también son mediados por la publicidad. En parte este fenómeno corresponde a los efectos del proceso de globalización y de la apertura comercial, que en cierta forma atentan contra la diversidad cultural en aras de la paradoja de un desarrollo económico mundial.'

Por ejemplo, el elevado número de personas que identificamos ahora con enfermedades cardiovasculares y neoplasias, se ha asociado al modo de vida individual y colectivo de la sociedad moderna, como el estrés, el abuso de alcohol y tabaco, el consumo de aditivos de alimentos procesados, el sedentarismo, la obesidad, la incorporación de materiales cancerígenos en las construcciones, asi como la contaminación del medio ambiente, del aire $y$ del agua, problemas que nos hablan, por si mismos, de que son insuficientes lasi estrategias de atención a la salud, tanto de los tomadores de decisiones en la materia como de las propias comunidades, y que enfrentamos más ahora un gran déficit en la cultura en salud.

Todos estos aspectos en conjunto, obligan al personal de Enfermería a retomar los enfoques Inter y transcultural en su ejercicio profesional, en el marco de los valores de la profesión.

\section{MARCO DE REFERENCIA PARA UN CUIDADO. CENTRADO EN LA CULTURA}

La palabra Cultura viene del latín, significa cultivar, hace referencia a los valores intrínsecos de la sociedad, a los instrumentos, las costumbres o instituciones de los grupos sociales y la utilización de tales medios. El término hace referencia al efecto que causan dichos elementos sobre el desarrollo y la expresión individual como el arte, la ciencia y la religión. (2) La cultura ha sido definida también como el fondo común de una nación o de un grupo social más restringido, compuesto principalmente de su lengua, su folklore, su arte y escala de valores, que se sustentan en su pasado histórico.

Todas las personas, por el hecho de nacer en una sociedad, son participes de una cultura, es decir de una configuración de comportamientos aprendidos y de sus resultados, cuyos elementos son compartidos por los miembros de esa sociedad, esto es, los valores, las formas de vivir y de pensar, cuya traducción cualitativa se evidencia en los sistemas de creencias y en los símbolos con que se expresan dichos componentes. La palabra contracultura refleja el rechazo, por un grupo minoritario, 
de los rasgos más característicos de la cultura nacional, así como la incorporación de nuevos valores con el objeto de exterminar a la sociedad establecida. Subcultura significa una distinción inducida por un grupo humano que aporta elementos propios sin ser contradictorios. ${ }^{3}$

La cultura de la salud como querer hacer y saber-hacer en el cultivo de la vida, atraviesa todo el continuo de la experiencia de cada hombre y generación. Como producción social especifica de un grupo, la cultura de la salud crea y recrea los "modos" de concebir, de parir y de nacer; de alimentar; de cuidar y de cuidarse; de trabajar; de festejar y hacer el duelo, de agonizar y de morir. Modo de criar, de educar y de aprender, de conocer, de envejecer, de divertirse o de divertir, de aburrirse y de aburrir. Modo de comunicar y expresarse. Es decir, todos los modos de hacer y de sentir que un grupo cultural asume como propios en el diario vivir, están dotados de representaciones, simbolos y sentidos que son comunes a cada grupo humano que los reviste de diferenciación.

La cultura de la salud en general, produce y reproduce sus modos, de manera espontánea en la vida cotidiana de la gente y en sus costumbres. En particular se manifiesta a través del arte, la ciencia, la tecnología, la ideologiá, la política, la cosmogonía, la educación, la comunicación, la publicidad, etc., y evoluciona a la par de la cultura general, con sus conocimientos, sus instituciones, sus sueños, ideas y deseos. ${ }^{4}$

Como cualquier hecho cultural, el cultivo de la vida y sus modos son, a la vez simbólicos y económicos. Ambos elementos interactúan entre sí de manera positiva - negativa y pueden garantizar o ser obstáculos de los deseos de calidad de vida o de muerte que posee un grupo social.

\section{SIGNIFICADO DE LA ENFERMERIAA TRANSCULTURAL}

El interés por las relaciones existentes entre las condiciones culturales y la salud es bastante antiguo y aunque en Enfermería se reconoce que para otorgar el cuidado competente debe tomarse en cuenta que existe una gran diversidad cultural, algunas Enfermeras no afrontan bien este compromiso. Por ejemplo mucho se insiste en que un buen cuidado es tratar a todos los pacientes por igual, en apariencia tratar "igualmente" a las personas suena bien, como un valor de justicia y que además está acorde con la democracia. Tratar a cada uno de igual manera, por supuesto que tiene un componente ético, pero también implica reconocer realmente las verdaderas diferencias que la cultura ocasiona en la conducta, en los valores y aun en la percepción del munndo de cada individuo.

En realidad un buen cuidado requiere que se acepte al paciente como individuo y en familia, en el contexto de su propia cultura. No es válido esperar que personas en crisis cambien sus creencias y conductas para ajustarse a la cultura del hospital. Sin embargo con frecuencia se insiste en lograr esto.

La teoría de los cuidados culturales de Madeleine Leininger trata sobre la importancia de otorgar cuidados de Enfermeria según sea la cultura del paciente, el conocimiento de la diversidad y la universalidad de la asistencia de Enfermería desde la óptica de esta autora, es de extraordinaria importancia por su enfoque holístico, en el que el entramado social, el entorno y el lenguaje, cobran una relevancia excepcional para poder descubrir y obtener un conocimiento basado en la cultura o sustentado en el mundo de la persona.

Leininger señaló, desde 1950, la existencia de varias áreas comunes de conocimiento y de interés científico teórico entre la Enfermería y la antropología, formuló diversos conceptos, teorias y principios de la Enfermería transcultural. Ha definido este término como una de las grandes áreas de la Enfermería, que se centra en el estudio y el análisis comparado de diferentes culturas y subculturas del mundo, desde el punto de vista; de sus valores asistenciales, de la expresión y convicciones sobre la salud enfermedad y de los modelos de conducta, siempre con el propósito de desarrollar una base de conocimientos científicos y humanísticos que permitan una práctica de la atención sanitaria especifica de una cultura y/ o universal. ${ }^{5}$

La autora establece también, la diferencia entre Enfermería transcultural e intercultural. La primera se refiere a los profesionales de Enfermería preparados y comprometidos en adquirir conocimiento y con un método práctico de actuación en el cuidada culturalmente competente. En cambio la Enfermeria intercultural se refiere a profesionistas que integran o aplican conocimientos antropológicos a la salud, sin comprometerse en el desarrollo de teorías - prácticas basadas en la investigación, dentro del propio campo disciplinar, que para este caso es la Enfermería. Cabe decir que la Enfermería transcultural contiene a la fase intercultural.

Esta teoría de la diversidad y universalidad de los cuidados culturales, se basa en la convicción de que las personas, usuarias de los servicios de salud, pueden ofrecer informa- 
ción especifica que oriente a los profesionales para que otorguen la clase de cuidados que desean o necesitan, más de acuerdo con sus valores culturales, y que finalmente asegure la calidad del servicio de Enfermeria. Ya que la cultura determina los patrones y estilos de vida que tienen influencia en las decisiones de las personas en lo que a salud se refiere, esta teoria ayuda a las Enfermeras a descubrir y documentar el mundo del paciente, para utilizar los puntos de vista émicos (perspectiva del sujeto de la atención) es decir, sus conocimientos y sus prácticas, en conjunción con una ética apropiada (perspectiva del profesional) como base para adoptar acciones y decisiones para el cuidado, más coherentes con los modos culturales.

Los cuidados culturales conforman la teoría integral de Enfermería más amplia que existe, ya que tiene en cuenta la totalidad, es decir la perspectiva holistica de la vida humana y de la existencia a lo largo del tiempo, incluyendo los factores sociales, la visión del mundo, la historia y los valores culturales, el contexto ambiental, el lenguaje y los modelos populares y profesionales.

La Enfermería transcultural enfatiza que los componentes objetivos del cuidado se enfocan hacia las características culturales del paciente y su familia y el cómo influyen en la salud y el cuidado. El ambiente del encuentro intercultural del cuidado, también influye en la forma como las personas interpretan lo que está ocurriendo y lo que expresan acerca de ellas mismas. También tiene importancia el tiempo que se dedica a cada paciente, lo cual está relacionado con las cargas de trabajo y el déficit de personal. ${ }^{6}$

En cambio, el componente subjetivo se enfoca en las características personales y culturales de la propia Enfermera y en la influencia de los conceptos especificos de la profesiön. No se puede brindar un cuidado culturalmente competente, sin tener una autoconciencia de nuestros valores, creencias y estilos de comunicación, porque estos tienen un poderoso impacto en el ejercicio profesional. La perspectiva cultural, es una combinación de conocimientos, actividades y conductas o habilidades que son interdependientes y requiere más que el simple conocimiento acerca de otro grupo étnico- cultural. Entre estos elementos se mencionan el respeto y la empatía con los pacientes, la flexibilidad, la comunicación efectiva y la negociación entre culturas.

La comunicación es el intercambio de mensajes y la creación de significados, todos nos comunicamos de maneras que son influenciadas por nuestra cultura, por ello, es importante tener conciencia de la manera en que nos comunicamos para poder ser más flexibles. Es muy común que un paciente considere a la Enfermera, insensible o incompetente, simplemente por las diferencias en la comunicación verbal y no verbal. Si el paciente piensa que la Enfermera es inadecuada, tendrá dificultạd para tenerle confianza y es posible que ignore sus sugerencias para el autocuidado. La Enfermera, por su parte puede no tener idea de que inconscientemente, envió tales mensajes.

Por ello, entre los aspectos importantes para ejercer la Enfermería transcultural, está el desarrollar habilidades para la comunicación no verbal, por ejemplo considerar el espacio personal que debe mediar entre Enfermera y paciente, el contacto visual, el estilo de comunicación, el tono de la voz y las concepciones del paciente respecto al "toque terapéutico".

Desde un punto de vista práctico, en la evaluación general del paciente con enfoque transcultural, debe imperar la apertura a nuevas experiencias y aprendizajes, es decir en el continuo del cuidado la Enfermera aprende de y con los pacientes. Es indispensable saber cual es el lenguaje del paciente, cuales son sus formas de comunicación no verbal, qué religión tiene y cómo la practica y si existe alguna prohibición religiosa para el tratamiento; qué clase de alimentos consume en su hogar, alergias y hábitos dietéticos. Cuales son las características culturales de la familia, es nuclear o extensa, existe o no el apoyo de los familiares en los momentos de crisis y en la rehabilitación. Se deben identificar las creencias sobre la enfermedad y los métodos y técnicas que se aplican para resolver los problemas de salud.

Siempre que la actitud de la Enfermera sea de interés profesional y de respeto por el paciente, será más factible vencer las barreras culturales y la desconfianza ante

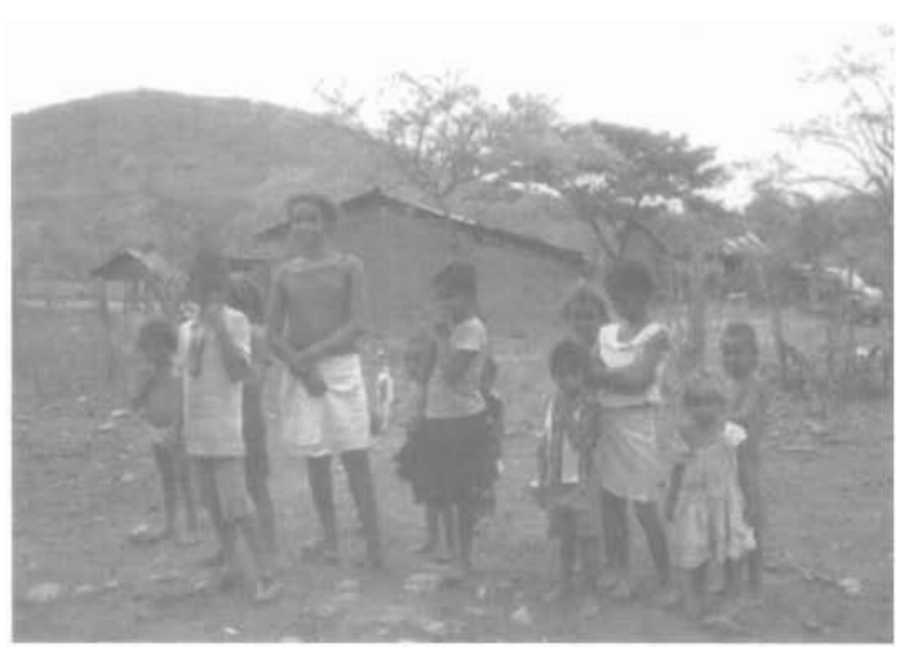


lo desconocido. Una regla general consiste en reforzar las creencias y las prácticas de salud positivas o neutras. Por ejemplo propiciar espacios para la oración cuando el paciente y sus familiares creen que esto ayudará a su curación, o que se lleven amuletos que lo protejan del "mal de. ojo". Asi también, ha de discutir con el paciente y la familia sobre las creencias o prácticas que son potencialmente dañinas, explicándoles cómo y porque ocurre esta.

\section{CONCLUSIONES}

Podemos afirmar que el cuidado que nos identifica en la perspectiva de la Enfermería transcultural es un cuidado reciproco, interactivo e integrador, en el cual las experiencias vividas y trascendentes comprenden la interacción entre personas totales, con sus sentimientos. pensamientos y expresiones. Las actitudes son producto de antecedentes familiares que influyen en la historia de vida de cada ser, por tal razón el ser es único e irrepetible y la oportunidad de interacción inter subjetiva, posibilita la integración y aceptación de sí mismo.

Interacción recíproca significa un encuentro de seres humanos diferentes, con sabidurías y mentalidades que cada uno ofrece en el encuentro, donde la persona del enfermo ofrece su ser total, sus conocimientos, sus observaciones y percepciones, consciente de que es un ser humano susceptible y vulnerable. El ambiente del cuidado debe favorecer la prioridad, el pudor y la unicidad de cada ser humano, sujeto reconocido del encuentro transcultural.
Gracias a las actividades auténticas de la Enfermera (0), conocimiento, reflexión, técnicas del cuidado y momentos especiales del mismo, se da esa interacción, en la que la persona ofrece su confianza, asi como su interés porque se le reestructure y reintegre como persona digna de apoyo, consideración, respeto y afecto.

\section{REFERENCIAS}

1. Frenk, Julio; Octavio Gómez Dantés. Globalización y salud en Observatono de la Salud. México: Fundación Mexicana para la salud; 1997, p.275-284

2. Feibleman, K. James. Diccionario de Filosofía. Caracas: Grijalbo; 1994, p. 79

3. Debasch, Charles y Duadet. Diccionario de términos politicos. Bogotá: Temis; 1995, p. 88

4. Pinto Afanador, Natividad. Cultura y cuidado de la salud. En Dimensiones del Cuidado, Facultad de Enfermería. Universidad Nacional de Colombia: Grupo de Cuidado; 1997, p. 47

5. Manrique; Maigualida; et. al. Madeleine Leininger Cuidados Cuiturales: Teoria de la diversidad y la universalidad. Monografla. Valencia, España: Universidad de Carabobo, Junio de 2003

6. Meleis, Isenberg, Koerner y Stern. Temas culturales en el cuidado de Enfermeria. Antioquia Medellín. Rev. RIEENF. $2002 ; 1$ (3): 12 .

\section{DIRECCIÓN PARA CORRESPONDENCIA:}

Ma. Teresa pérez Rodriguez : pisis_tete@yahoo.com.mx

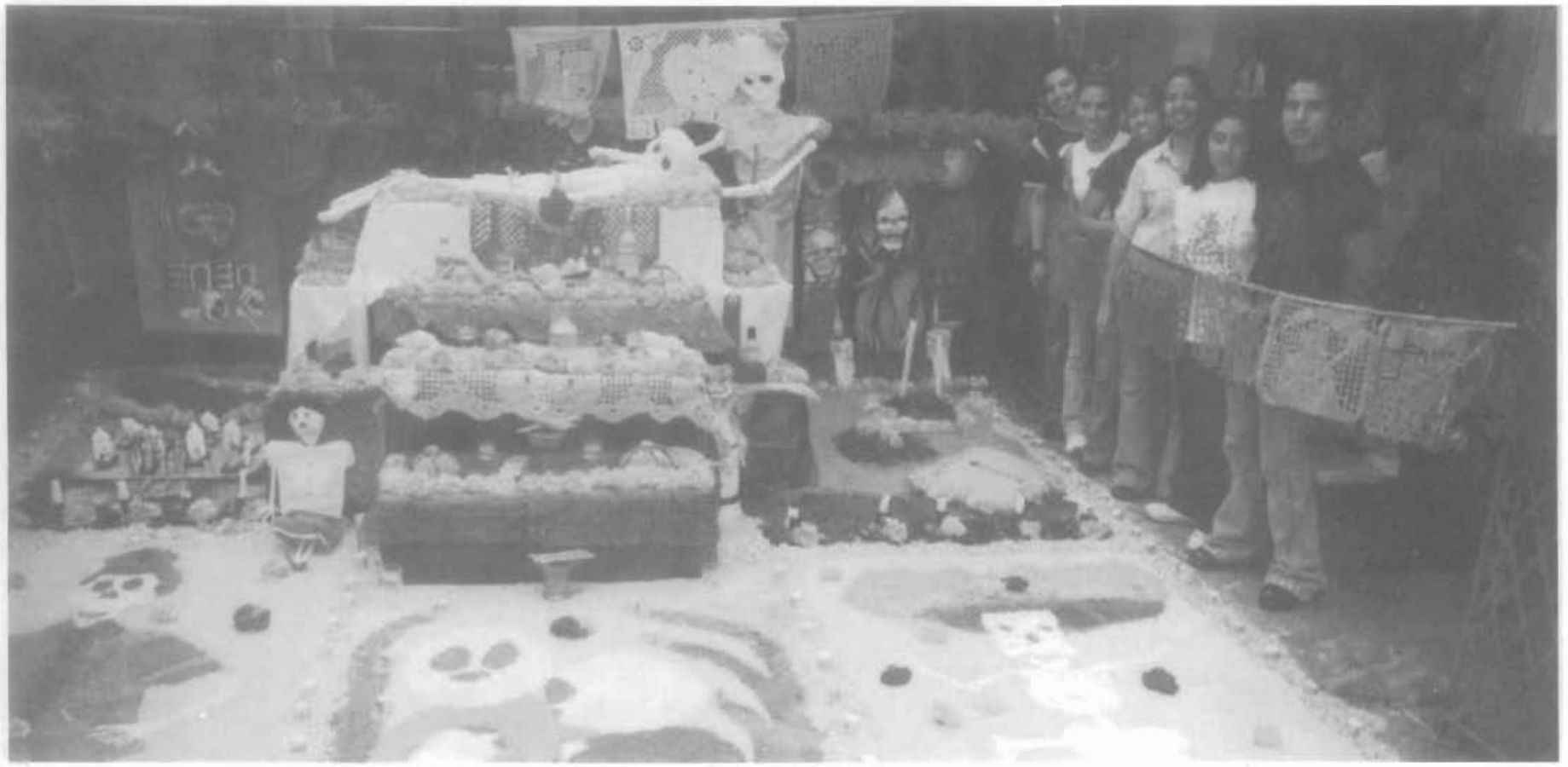

